\title{
Tripterygium glycoside suppresses epithelial-to-mesenchymal transition of diabetic kidney disease podocytes by targeting autophagy through the mTOR/Twist1 pathway
}

\author{
MEI TAO ${ }^{1-3}$, DANNA ZHENG ${ }^{2,3}$, XUDONG LIANG $^{2,3}$, DIANDIAN WU ${ }^{2,3}$, \\ KANG HU ${ }^{2,3}$, JUAN JIN ${ }^{2,3^{*}}$ and QIANG $\mathrm{HE}^{2,3^{*}}$ \\ ${ }^{1}$ Department of Nephrology, The Affiliated Hospital of Hangzhou Normal University, Hangzhou, Zhejiang 310015; \\ ${ }^{2}$ Department of Nephrology, Zhejiang Provincial People's Hospital and Affiliated People's Hospital, \\ Hangzhou Medical College, Hangzhou, Zhejiang 310014; ${ }^{3}$ Department of Nephrology, \\ Chinese Medical Nephrology Key Laboratory, Zhejiang Provincial People's Hospital and \\ Affiliated People's Hospital, Hangzhou, Zhejiang 310014, P.R. China
}

Received May 29, 2020; Accepted March 25, 2021

DOI: $10.3892 / \mathrm{mmr} .2021 .12231$

\begin{abstract}
Tripterygium glycoside (TG) is a traditional Chinese medicine extract with immunosuppressive, anti-inflammatory and anti-renal fibrosis effects. Epithelial-mesenchymal transition (EMT) and cell apoptosis are considered to be the major cause of podocyte injury in diabetic kidney disease (DKD). However, it remains unknown as to whether TG is able to alleviate podocyte injury to prevent DKD progression. Therefore, the present study aimed to clarify the podocyte protective effects of TG on DKD. TG, Twist1 small interfering RNA (siRNA) and Twist1 overexpression vector were added to DKD mouse serum-induced podocytes in vitro. Autophagic and EMT activities were evaluated by immunofluorescence staining and western blot analysis. Apoptotic activity was evaluated by Annexin V-FITC/PI flow cytometric analysis. The results revealed that after treatment with DKD mouse serum, autophagy was decreased, whereas EMT and apoptotic rate were increased, in podocytes. In addition, Twist1 expression was increased in DKD-induced podocytes. Furthermore, following
\end{abstract}

Correspondence to: Dr Qiang He or Dr Juan Jin, Department of Nephrology, Zhejiang Provincial People's Hospital and Affiliated People's Hospital, Hangzhou Medical College, 158 Shangtang Road, Hangzhou, Zhejiang 310014, P.R. China

E-mail: qianghe1973@126.com

E-mail: lang_018@163.com

${ }^{*}$ Contributed equally

Abbreviations: TG, tripterygium glycoside; 3BDO, 3-benzyl-5((2-nitrophenoxy) methyl)-dihydrofuran-2(3H)-one; EMT, epithelialmesenchymal transition; DKD, diabetic kidney disease; ESRD, end-stage renal disease

Key words: podocytes, autophagy, diabetic kidney disease, tripterygium glycoside, epithelial-mesenchymal transition
Twist1-small interfering RNA transfection, the DKD-induced podocyte EMT and apoptotic rate were markedly reduced, indicating that Twist 1 may be a promising therapeutic target for DKD. The present results also revealed that overexpression of Twist1 increased podocyte apoptosis, although this was decreased after TG treatment, indicating that TG may exhibit a protective effect on podocytes by inhibiting the Twist1 signaling pathway. After the addition of 3-benzyl-5-((2-nitrophenoxy) methyl)-dihydrofuran-2(3H)-one, an activator of mTORC1, the effects of TG on podocyte EMT, apoptosis and the autophagy were reversed. These findings indicated that TG may alleviate EMT and apoptosis by upregulating autophagy through the mTOR/Twist1 signaling pathway in DKD.

\section{Introduction}

Diabetic kidney disease (DKD) is a chronic renal condition and the most common cause of end-stage renal disease (ESRD) worldwide (1). Controlling blood glucose and blood pressure levels can appropriately delay the onset of ESRD, and several hyperglycemic drugs with renal protection have been reported, including sodium-glucose co-transporter- 2 inhibitors and glucagon-like peptide 1 receptor agonists (2). However, due to the high morbidity and complicated pathogenesis of CKD, further studies on novel drugs and their mechanisms of action are required to prevent the progression of DKD.

Epithelial-mesenchymal transition (EMT) is a process where epithelial cells acquire mesenchymal properties, as characterized by increased expression of vimentin and $\mathrm{N}$-cadherin, and decreased expression of specific epithelial cell markers, such as nephrin, zonula occludens-1 (ZO-1) and E-cadherin. Glomerular podocytes are essential for the correct function of the glomerular filtration barrier. Podocyte injury and loss contribute to the progression of DKD (3). Previous studies have proposed that podocytes undergo EMT stimulated by high glucose $(4,5)$, transforming growth factor- $\beta$ (TGF- $\beta$ ) (6-9), adriamycin (8) and homocysteine (10), leading to podocyte detachment or dysfunction, which ultimately results in 
glomerular filtration dysfunction. Numerous studies have reported that podocytes are phenotypically altered in the early stages of a rodent model of DKD induced by high glucose and TGF- $\beta$, with increased mesenchymal markers (desmin) and decreased epithelial markers (nephrin) $(5,7,9)$. In addition to the findings in animal studies, a marked reduction in nephrin and ZO-1 expression has been observed in the glomeruli of patients with DKD (5,11-12). Furthermore, podocyte EMT has been reported to participate in the loss of podocytes in CKD through induction of podocyte detachment or apoptosis (11). Accumulating evidence suggests that EMT may be a potential pathway that contributes to the progression of DKD. Therefore, it may be utilized as a novel route and potential drug target for therapeutic interventions in DKD.

Autophagy is a highly regulated lysosomal pathway involved in cytoplasm recycling, and removal of excess or damaged organelles. It is essential for cell survival, differentiation, development and homeostasis. Changes in autophagy are detected by microtubule-associated protein 1 light chain 3 (LC3) and sequestosome 1 (SQSTM1/P62). LC3 is located on the autophagosome membrane and consists of cytoplasmic LC3 I and membrane-bound LC3 II (13). Previous studies have reported that autophagy is a protective mechanism of podocytes, and autophagy dysfunction is the major risk factor for podocyte injury, including apoptosis (14) and EMT (15).

Twist 1 regulates the occurrence of cellular EMT by controlling the transcription of EMT-associated genes through promoter activation or repression; it downregulates epithelial phenotype-related genes, such as E-cadherin, and upregulates mesenchymal cell phenotype-related genes, such as vimentin (16). Twist1 has been shown to promote EMT in endometrial and liver cancer through vimentin regulation $(17,18)$, indicating that it is a key gene in EMT and cancer. While the specific role of Twist1 in DKD remains unclear, Qiang and He (19) reported that autophagy deficiency may inhibit the degradation of Twist1 through SQSTM1/p62 accumulation to promote cellular EMT, thus proposing a novel mechanism for the regulation of autophagy and EMT.

Tripterygium glycoside (TG) is a fat-soluble mixture (composed of diterpene lactone, alkaloid and triterpenoid) extracted from the root xylem of Tripterygium wilfordii Hook F, which has anti-inflammatory, immunosuppressive, immunomodulatory and anti-tumor effects (20). TG has been reported to markedly attenuate renal injury and to regulate immune-inflammatory responses in DKD animal models $(21,22)$. The present study aimed to explore the effect of TG on podocyte autophagy, apoptosis and EMT. It was hypothesized that TG could restore autophagy to alleviate EMT and apoptosis via the mTOR/Twist1 signaling pathway, resulting in the improvement of DKD.

\section{Materials and methods}

Patient selection and renal biopsies. All patients with DKD were diagnosed based on renal biopsies carried out at the Department of Nephrology, Zhejiang Provincial People's Hospital (Hangzhou, China). The patients were selected using the Mayo Clinic/Renal Pathology Society Consensus Report on Pathological Classification, Diagnosis, and Reporting of GN (23). The demographic and clinical data of these patients, including age, systolic blood pressure (SBP), blood urea nitrogen (BUN), serum creatinine (Scr), fasting plasma glucose (FPG), glycosylated hemoglobin A1c (HbA1c), low-density lipoprotein cholesterol (LDL-C) and high-density lipoprotein cholesterol (HDL-C) were obtained. A total of 20 patients with DKD (11 male patients, nine female patients; age range, 36-87 years) and 10 normal human controls (six men, four women; age range, 22-44 years) were enrolled in the study. All protocols concerning the use of patient samples in the present study were approved by the Human Subjects Committee of Zhejiang Provincial People's Hospital. Written informed consent was obtained from all donors.

Animals. A total of 16 mice, including male spontaneous diabetic nephropathy mice (C57BL/KsJ db/db; n=12; age, 8 weeks; weighing, 16-20 g) and male healthy control mice (C57BL/KsJ db/m; n=4; age, 8 weeks; weighing, 16-20 g) were provided by Changzhou Cavans Laboratory Animal Co., Ltd. [license no. SCX (Su) 2016-0010]. The mice were housed in a pathogen-free facility under a 12-h light/dark cycle, with $50-65 \%$ humidity at $22-25^{\circ} \mathrm{C}$. Mice were supplied with continuous access to drinking water and a normal diet, and were observed weekly. At 13 weeks of age, blood samples were collected from the tail vein of mice (total volume collected, $1.5 \mathrm{ml}$; volume collected from each mouse, $\sim 100 \mu \mathrm{l}$ ), and the blood samples were coagulated for 20-30 min before being centrifuged at $2,000 \mathrm{x}$ g for $20 \mathrm{~min}$ at $4^{\circ} \mathrm{C}$. The serum was removed from the centrifuged samples and was stored at $-20^{\circ} \mathrm{C}$ until use. All sera used were thawed and heat-inactivated at $56^{\circ} \mathrm{C}$ before being used in subsequent cell culture experiments (24). At the end of the experimental period, the mice $(n=16)$ were sacrificed by cervical dislocation. Death was confirmed by checking whether the heart and respiration of the mice had stopped completely and the pupils were dilated. The experiments were carried out in strict accordance with the National Institutes of Health Guide for the Care and Use of Laboratory Animals (25). All protocols involving animals were approved by the Institutional Animal Care and Use Committee of Zhejiang Provincial People's Hospital.

Cell culture. The mouse podocyte MPC5 cell line was obtained from the Shanghai Institutes for Biological Sciences, Chinese Academy of Sciences. Podocytes were cultured and expanded at $33^{\circ} \mathrm{C}$ in RPMI-1640 medium (HyClone; Cytiva) supplemented with 10\% FBS (cat. no. SH30084.03; HyClone; Cytiva), a low concentration of glucose (11 nM D-glucose) and $20 \mathrm{U} / \mathrm{ml} \gamma$-IFN (cat. no. CSB-E04578m; Cusabio Technology LLC). Podocyte MPC5 cells were aspirated and passaged at a ratio of 1:4. Podocytes were cultured at $37^{\circ} \mathrm{C}$ and $5 \% \mathrm{CO}_{2}$ for maturation. Before podocytes were passaged, type IV collagen $\left(1.5 \mathrm{ml} / 25 \mathrm{~cm}^{2}\right.$; cat. no. CSB-E08884m; Cusabio Technology LLC) was placed in the culture flask and cells were incubated for $1 \mathrm{~h}$. After washing the bottom of the culture flask with PBS $\left(3 \mathrm{ml} / 25 \mathrm{~cm}^{2}\right)$, the cells were aspirated and passaged at a ratio of 1:2. To induce differentiation, MPC5 cells were grown under restrictive conditions at $37^{\circ} \mathrm{C}$ for 10 days, then MPC5 cells were treated simultaneously for $24 \mathrm{~h}$ with $0.2 \%$ FBS in $5 \mathrm{mM}$ D-glucose RPMI-1640. Mature and differentiated podocytes were collected and seeded uniformly in a 6-well 
plate, and the concentration of podocytes was adjusted to $1 \times 10^{6}$ cells $/ \mathrm{ml}$. A total of $0.5 \mathrm{ml}$ podocyte suspension and $1.5 \mathrm{ml}$ RPMI-1640 medium containing 10\% FBS were added to each well (6-well plate) and were cultured at $37^{\circ} \mathrm{C}$ in a $5 \% \mathrm{CO}_{2}$ incubator. MPC5 cells were treated with $10 \% \mathrm{DKD}$ mice serum $(\mathrm{C} 57 \mathrm{BLKS} / \mathrm{J} \mathrm{db} / \mathrm{db})$ or $10 \%$ control mice serum (C57BLKS $/ \mathrm{J} \mathrm{db} / \mathrm{m}$ ) at $37^{\circ} \mathrm{C}$ for $24 \mathrm{~h}$.

Drugs. TG (cat. no. 14002219121) was purchased from Zhejiang DND Pharmaceutical Co., Ltd. Podocytes were treated with TG $(1.25 \mu \mathrm{g} / \mathrm{ml})$ at $37^{\circ} \mathrm{C}$ for $72 \mathrm{~h}$. The effective concentration of $\mathrm{TG}$ was verified in our preliminary experiments (26). 3-benzyl-5-((2-nitrophenoxy) methyl)-dihydrofuran-2(3H)-one (3BDO; cat. no. S8317) was purchased from Selleck Chemicals. Podocytes were treated with 3BDO $(60 \mu \mathrm{M})$ at $37^{\circ} \mathrm{C}$ for $24 \mathrm{~h}(27)$.

ELISA. Levels of p62, Twist1 and E-cadherin in human serum samples were detected using p62 ELISA kit (cat. no. NBP2-61300; Novus Biologicals, LLC), Twist1 ELISA kit (cat. no. NBP3-06809; Novus Biologicals, LLC) and E-cadherin ELISA kit (cat. no. KA0433; Abnova), respectively. Serum N-cadherin and vimentin levels were detected using N-cadherin ELISA kit (cat. no. CSB-E09718h; Cusabio Technology LLC) and vimentin ELISA kit (cat. no. CSB-E08982h; Cusabio Technology LLC). All ELISA kits were used according to the manufacturer's protocols.

Urine protein content determination. Mice $(n=16)$ were raised to 13 weeks of age in a metabolic cage. Urine was then collected for $24 \mathrm{~h}$ and the protein levels in urine were determined using a BCA kit (cat. no. P0010; Beyotime Institute of Biotechnology). Protein concentrations were calculated from the standard curve and the sample volume used.

Immunofluorescence staining. Cells cultured on coverslips were washed three times with PBS and fixed with $4 \%$ paraformaldehyde for $15 \mathrm{~min}$ at room temperature. Cells were then treated with $0.1 \%$ Triton $\mathrm{X}-100$ for $10 \mathrm{~min}$ and blocked with normal goat serum (cat. no. C0265; Beyotime Institute of Biotechnology) for $30 \mathrm{~min}$ at room temperature. Cells were then incubated with specific primary antibodies against LC3 (1:50; cat. no. 14600-1-AP; ProteinTech Group, Inc.) and vimentin (1:50; cat. no. bs-8533R; BIOSS) at $4^{\circ} \mathrm{C}$ overnight in a wet box. Subsequently, the samples were incubated with the corresponding secondary antibody for $1 \mathrm{~h}$ at room temperature [Alexa Fluor 594-conjugated AffiniPure Goat Anti-Rabbit IgG (H+L); 1:100; cat. no. 111585003; Jackson ImmunoResearch Laboratories, Inc.; and Alexa Fluor 488-conjugated AffiniPure Goat Anti-Rabbit IgG (H+L); 1:100; cat. no. 111545003; Jackson ImmunoResearch Laboratories, Inc.]. Finally, cells were stained with DAPI hydrochloride to visualize the nuclei. Slides were visualized under a fluorescence microscope (DM6000B; Leica Microsystems, Inc.).

Western blot analysis. Cells were washed three times with ice-cold PBS and lysed with cell lysis RIPA buffer (cat. no. P0013B; Beyotime Institute of Biotechnology) for $20 \mathrm{~min}$. The cell pellet and lysate were collected and centrifuged at $14,000 \mathrm{x} \mathrm{g}$ for $4 \mathrm{~min}$ at $4^{\circ} \mathrm{C}$. The supernatant was collected and the protein concentration was determined using a BCA kit. An appropriate volume of $5 \mathrm{X}$ loading mix was added to the samples and heated for $5 \mathrm{~min}$ at $100^{\circ} \mathrm{C}$ to denature the proteins. Samples and pre-stained markers were added as required. The protein samples $(30 \mu \mathrm{g})$ were mixed with loading buffer and subjected to SDS-PAGE on $12 \%$ gels. The electrically-transformed PVDF membrane was taken out and washed with TBS containing 0.1\% Tween-20 (TBST) and blocked with $5 \%$ milk in TBST on a shaker at room temperature for $1 \mathrm{~h}$. Subsequently, primary antibodies against E-cadherin (1:1,000; cat. no. 20874-1-AP; ProteinTech Group, Inc.), N-cadherin (1:1,000; cat. no. 22018-1-AP; ProteinTech Group, Inc.), Twist1 (1:500; cat. no. 25465-1-AP; ProteinTech Group, Inc.), LC3 (1:1,000; cat. no. 14600-1-AP; ProteinTech Group, Inc.); phosphorylated (p)-mTOR (1:1,000; cat. no. 5536T; Cell Signaling Technology, Inc.), p62 (1:1,000; cat. no. 184201-AP; ProteinTech Group, Inc.); mTOR (1:1,000; cat. no. 20657-1-AP; ProteinTech Group, Inc.) and $\beta$-actin (1:3,000; cat. no. 4970; Cell Signaling Technology, Inc.) were added and incubated at $4^{\circ} \mathrm{C}$ overnight. After washing the membranes with PBS containing 0.1\% Tween-20 (PBST) three times, the membranes were incubated with a horseradish peroxidase-labeled secondary antibody (1:5,000; cat. no. A0208; Beyotime Institute of Biotechnology) diluted in $5 \%$ milk/PBST for $1 \mathrm{~h}$ at room temperature on a shaker. The PVDF membrane was exposed to the luminescent reagent for coloring, and after 1.5-2 min, it was observed using a gel imaging system (ChemiDoc $^{\mathrm{TM}}$ XRS+; Bio-Rad Laboratories, Inc.). ImageJ software (version $1.49 \mathrm{p}$; National Institutes of Health) was used to calculate the gray value and analysis of the protein bands.

Flow cytometry. The supernatant treated as aforementioned was aspirated and transferred to a microtube for storage. Cells were washed twice with PBS and collected. Cells were then digested with $0.25 \%$ trypsin (without EDTA) at $37^{\circ} \mathrm{C}$ for 2-3 min. A pre-mixed 1X Annexin V Binding Solution was added to the cell suspension at a final concentration of $1 \times 10^{6}$ cells $/ \mathrm{ml}$. A total of $5 \mu \mathrm{l}$ Annexin V-FITC conjugate and $5 \mu \mathrm{l}$ propidium iodide (cat. no. KGA108; Nanjing KeyGen Biotech Co., Ltd.) were then added to the cell suspension and incubated for $10 \mathrm{~min}$ at room temperature in the dark. Finally, flow cytometry (cytoFLEX; Beckman Coulter, Inc.) was conducted within $1 \mathrm{~h}$ of adding $400 \mu \mathrm{l} 1 \mathrm{X}$ Annexin V Binding Solution. The apoptotic rate of cells (including early and late apoptotic cells) was analyzed using CytExpert version 2.0 software (Beckman Coulter, Inc.).

Twist1 small interfering RNA (siRNA) synthesis and transfection. siRNA sequences [three specific interference sequences and one negative control (NC) sequence] were designed and synthesized (GenScript) to target mouse Twist1. MPC5 cells were inoculated into a six-well plate at $5 \times 10^{5}$ cells/well and cultured at $37^{\circ} \mathrm{C}$ for $24 \mathrm{~h}$ in a $5 \% \mathrm{CO}_{2}$ incubator. After cell attachment, transfection was performed with Lipofectamine $^{\circledR} 2000$ (Invitrogen; Thermo Fisher Scientific, Inc.) according to the manufacturer's instructions. Briefly, one Eppendorf(EP) tube(A tube) containing $5 \mu$ Lipofectamine 2000 diluted with $250 \mu \mathrm{l}$ Opti-MEM (Thermo Fisher Scientific, Inc.) was incubated at room temperature for $5 \mathrm{~min}$, whereas another 
EP tube (B tube) contained $2 \mu \mathrm{l}$ siRNA diluted with $250 \mu 1$ Opti-MEM. The A and B tubes were gently mixed and allowed to stand at room temperature for $20 \mathrm{~min}$. The siRNA-Lipofectamine 2000 mixture was then added to a six-well plate ( $5 \times 10^{5}$ cells/well) containing $1.5 \mathrm{ml}$ complete medium. The medium was changed after 4-6 h of transfection. Subsequent experiments were performed at $48 \mathrm{~h}$ post-transfection. The siRNA sequences were as follows: siRNA-NC (sense, 5'-UUC UCCGAACGUGUCACGUTT-3' and antisense, 5'-ACGUGA CACGUUCGGAGAATT-3'), siRNA1-Twist1 (sense, 5'-CCU CUGCAUUCUGAUAGAATT-3' and antisense, 5'-UUCUAU CAGAAUGCAGAGGTT-3'), siRNA2-Twist1 (sense, 5'-GUG UCUAAAUGCAUUCAUATT-3' and antisense, 5'-UAUGAA UGCAUUUAGACACTT-3') and siRNA3-Twist1 (sense, 5'-GGU ACAUCGACUUCCUGUATT-3' and antisense, 5'-UAC AGGAAGUCGAUGUACCTT-3').

Construction of Twistl overexpression (OE) plasmid vector. Plasmids pcDNA3.1-Hygro(+) and pUC57-Mus Twist1 (both from GenScript) were double digested with XhoI and NotI (both from Takara Bio, Inc.) at $37^{\circ} \mathrm{C}$ overnight, and the vector and fragment were recovered and purified on an agarose gel by double digestion. The recovered and purified target fragment was ligated with the recovered and purified vector at $4^{\circ} \mathrm{C}$ overnight. The thawed DH5 $\alpha$ competent cells and plasmid were thoroughly mixed and stabilized on ice for $30 \mathrm{~min}$. Then, the mixture was put in a water tank at $42^{\circ} \mathrm{C}$ for 90 sec. Subsequently, $400 \mu 1$ Luria-Bertani medium ( $1 \%$ tryptone, $0.5 \%$ yeast extract, $1 \% \mathrm{NaCl}$; purchased from Merck KGaA) was added, and the culture was shaken at $37^{\circ} \mathrm{C}$ for $1 \mathrm{~h}$. Subsequently, $1 \mathrm{ml}$ Luria-Bertani medium broth was added to the mixture, which was then incubated at $37^{\circ} \mathrm{C}$ for $1 \mathrm{~h}$. The transformed DH5 $\alpha$ competent cells were plated onto Luria Bertani-containing Petri dishes with $100 \mu \mathrm{g} / \mathrm{ml}$ ampicillin and incubated overnight at $37^{\circ} \mathrm{C}$. Several monoclonal colonies were selected from the Petri dish, inoculated in ampicillin-resistant Luria Bertani culture medium, cultured at $37^{\circ} \mathrm{C}$ at overnight and subjected to colony PCR. A single colony was carefully picked up and placed in the EP tube

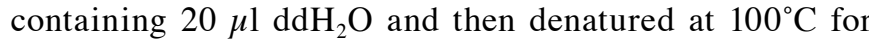
2 min. A $1 \mu \mathrm{l}$ suspension was used as a template in PCR reactions. PCR reactions were performed in a $20 \mu \mathrm{l}$ volume containing 2.5 $\mu \mathrm{l}$ 10X Taq DNA Polymerase buffer, $2 \mu \mathrm{ldNTP}$, $0.5 \mu \mathrm{M}$ of each primer and $0.5 \mu \mathrm{l}$ Taq DNA polymerase. The reaction protocols were as follows: Initial denaturation at $94^{\circ} \mathrm{C}$ for $10 \mathrm{~min} ; 30 \mathrm{cycles}$ of $94^{\circ} \mathrm{C}$ for $30 \mathrm{sec}, 60^{\circ} \mathrm{C}$ for $30 \mathrm{sec}$ and $72^{\circ} \mathrm{C}$ for $1 \mathrm{~min}$. The primer sequences were as follows: CMV-Forward: 5'-CGCAAATGGGCGGTAGGCGTG-3' and BGH-Reverse: 5'-TAGAAGGCACAGTCGAGG-3'. Cell transfection was performed using Lipofectamine 2000. MPC5 cells were seeded into a six-well plate at $5 \times 10^{5}$ cells/well and cultured overnight to $90 \%$ coverage, then transfected with Twist1 overexpression vector $(4 \mu \mathrm{g})$ or empty vector at $37^{\circ} \mathrm{C}$ for $72 \mathrm{~h}$. Untransfected cells were used as the control cells. Cells transfected with empty vector were used as NC cells.

Statistical analysis. All experiments were repeated three times. All statistical analyses were performed with SPSS 19.0 software (IBM Corp.). Data are presented as the mean \pm standard deviation. Comparisons between multiple groups were analyzed by one-way ANOVA followed by Tukey's post-hoc test and comparisons between two groups were analyzed by unpaired Student's t-test. $\mathrm{P}<0.05$ was considered to indicate a statistically significant difference.

\section{Results}

Determination of serum E-cadherin, $N$-cadherin, vimentin, p62 and Twistl levels in patients with DKD. Serum samples from healthy controls $(n=10)$ and patients with DKD $(n=20)$ were collected for biochemical index detection. According to the urinary albumin to creatinine ratio (UACR), patients with DKD were divided into two groups: DKD-microalbuminuria (micro; UACR, 30-300 mg/g) and DKD-macroalbuminuria (macro; UACR, $>300 \mathrm{mg} / \mathrm{g}$ ). The demographic and clinical data for these patients are listed in Table SI. The results indicated that age, SBP, BUN, Scr, FPG and HbA1c were significantly higher, whereas HDL-C was significantly decreased in the macro group compared with the control group. Similarly, compared with the control group, the age, SBP, FPG, and HbAlc of the micro group were significantly increased, whereas HDL-C was significantly decreased. ELISA was used to detect the levels of autophagy and EMT-related proteins. In patients with DKD, a significant decrease in serum E-cadherin levels, and a significant increase in serum $\mathrm{N}$-cadherin, vimentin, p62 and Twist 1 levels were observed compared with those in the healthy controls (Fig. 1).

Quantitative determination of urinary protein in mice. The present study raised 8 -week-old mice until they were 13 weeks of age. To confirm that the mice had diabetic nephropathy, urine was collected for $24 \mathrm{~h}$ to determine the protein content. The results revealed that the 24-h urinary protein content in mice with diabetic nephropathy was significantly higher than that in control mice (Fig. 2), indicating that the model was reliable and could be used for serum preparation.

Effects of Twistl on DKD-induced podocyte EMT and apoptosis. Since Twist1 is known to serve a vital role in EMT, the present study investigated whether Twist1 was able to regulate EMT and apoptosis of podocytes induced by serum of DKD mice. Three interference sequences were designed for cell transfection of MPC5 cells, and the most effective siRNA (siRNA2-Twist1) was selected for subsequent experiments (Fig. 3A and B). The immunofluorescence results showed that, compared with those in the control group, the protein expression levels of vimentin were significantly increased in the DKD group, but were inhibited after siRNA2-Twist1 transfection (Fig. 4A and B). The western blotting results revealed that the protein expression levels of Twist1 and $\mathrm{N}$-cadherin were significantly increased, whereas the expression levels of E-cadherin were significantly decreased in the DKD group compared with those in the control group. After siRNA2-Twist1 transfection, compared with those in the DKD + siRNA-NC group, the expression levels of Twist1 and N-cadherin were significantly decreased, whereas the expression levels of E-cadherin were markedly increased (Fig. 4C and D). Both immunofluorescence and western blotting results demonstrated that silencing Twist1 alleviated DKD-induced podocyte EMT. Furthermore, the results of flow cytometry demonstrated that, 

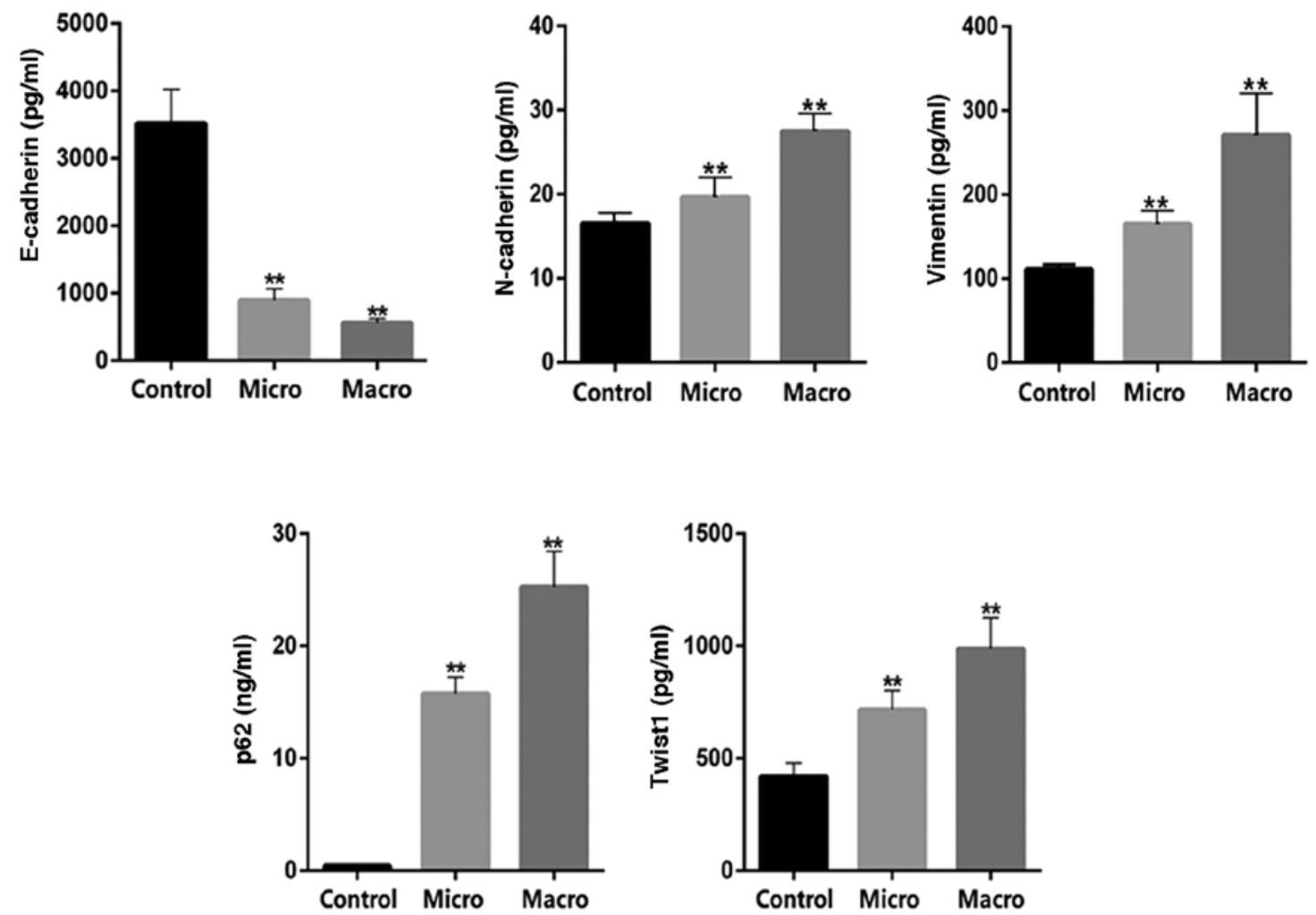

Figure 1. Autophagy and epithelial-mesenchymal transition analysis in patients with DKD. Control, healthy participants (n=10); micro, patients with DKD and microalbuminuria ( $\mathrm{n}=10$; UACR, 30-300 mg/g); macro, patients with DKD and macroalbuminuria ( $\mathrm{n}=10$; UACR, $>300 \mathrm{mg} / \mathrm{g}$ ). Data analysis was performed by one-way ANOVA followed by Tukey's post-hoc test. Data are presented as the mean \pm standard deviation. ${ }^{* *} \mathrm{P}<0.01$ vs. control. DKD, diabetic kidney disease; UACR, urinary albumin to creatinine ratio.

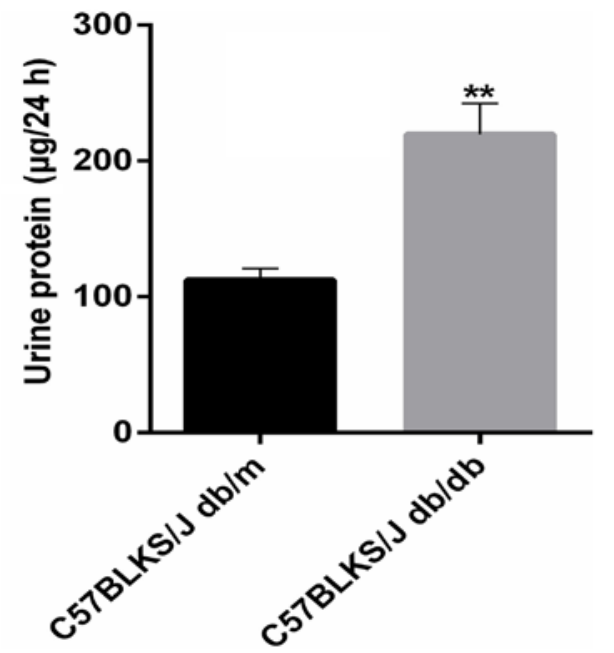

Figure 2. Urine protein content $(24 \mathrm{~h})$ from mice with diabetic nephropathy and control mice. C57BLKS/J db/m, normal control mice $(\mathrm{n}=4)$; C57BLKS/J $\mathrm{db} / \mathrm{db}$, mice with diabetic kidney disease $(\mathrm{n}=12)$. Data analysis was performed by unpaired Student's t-test. Data are presented as the mean \pm standard deviation. ${ }^{* *} \mathrm{P}<0.01$ vs. C57BLKS/J db/m.

after siRNA-Twist1 transfection, the apoptosis of podocytes in the DKD group was inhibited compared with those in the DKD + siRNA-NC group (Fig. 4E and F), which indicated that silencing Twist1 could reduce podocyte apoptosis and EMT.

TG inhibits DKD-induced podocyte apoptosis through Twistl. After determining the role of Twist1 in EMT and apoptosis, the present study aimed to determine the effect of TG on Twist 1 by constructing a Twist1 OE vector. The expression of Twist1 increased significantly after transfection with Twist1 OE vector in MPC5 cells, indicating that the Twist1 OE vector was successfully constructed (Fig. 5A and B). As shown in Fig. 6A and B, flow cytometry revealed that, compared with that in the control group, the apoptotic rate was significantly increased in podocytes in the DKD group. There was no significant difference in the rate of apoptosis between the DKD group and the DKD + Vector group, indicating that transfection with an empty vector did not affect the apoptosis of podocytes. Furthermore, compared with that in the DKD + Vector group podocyte apoptosis was significantly increased in the DKD + Twist1 OE group, indicating that Twist1 overexpression increased podocyte apoptosis. In the DKD + $\mathrm{TG}+$ Vector group, the apoptotic rate was inhibited by TG compared with that in the DKD + Vector group, indicating that TG could inhibit podocyte apoptosis. In addition, compared with that in the DKD + TG + Vector group, the apoptotic rate of podocytes was significantly increased in the DKD + TG + Twist1 OE group. These results indicated that TG inhibited podocyte apoptosis through Twist1.

TG alleviates podocyte EMT and apoptosis by upregulating autophagy through the mTOR/Twistl signaling pathway. 3BDO, a mTOR activator, was applied to confirm that TG alleviates podocyte EMT and apoptosis through the autophagy pathway. As shown in Fig. 7A and B, the fluorescent signals of vimentin were significantly increased in the DKD group, but 
A

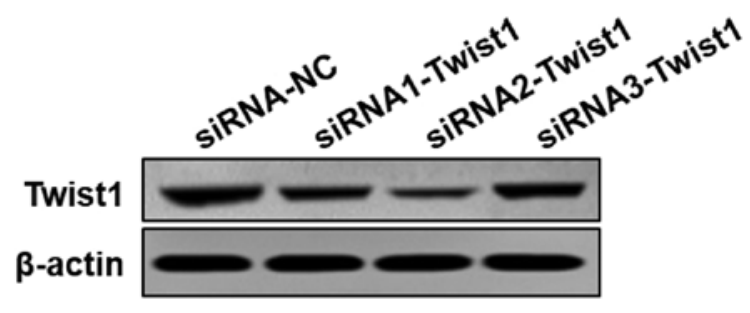

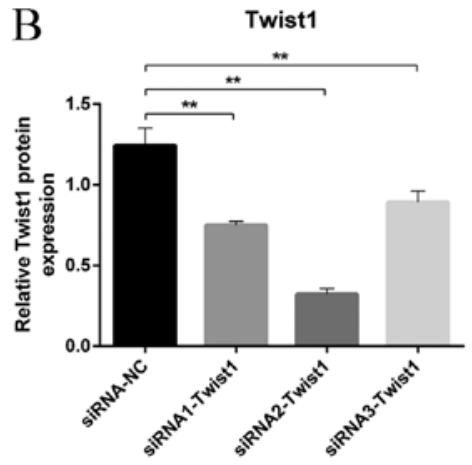

Figure 3. Twist1 interference screening. (A) Representative western blots of Twist1 expression in the different groups. (B) Densitometric analysis of Twist1 expression, as determined by western blotting. siRNA-NC, cells transfected with siRNA-NC for $72 \mathrm{~h}$; siRNA1/2/3-Twist1, cells transfected with siRNA1/2/3-Twist1 for $72 \mathrm{~h}$. Data are presented as the mean \pm standard deviation. Data analysis was performed by one-way ANOVA followed by Tukey's post-hoc test. ${ }^{* * *} \mathrm{P}<0.01$. siRNA, small interfering RNA; NC, negative control.
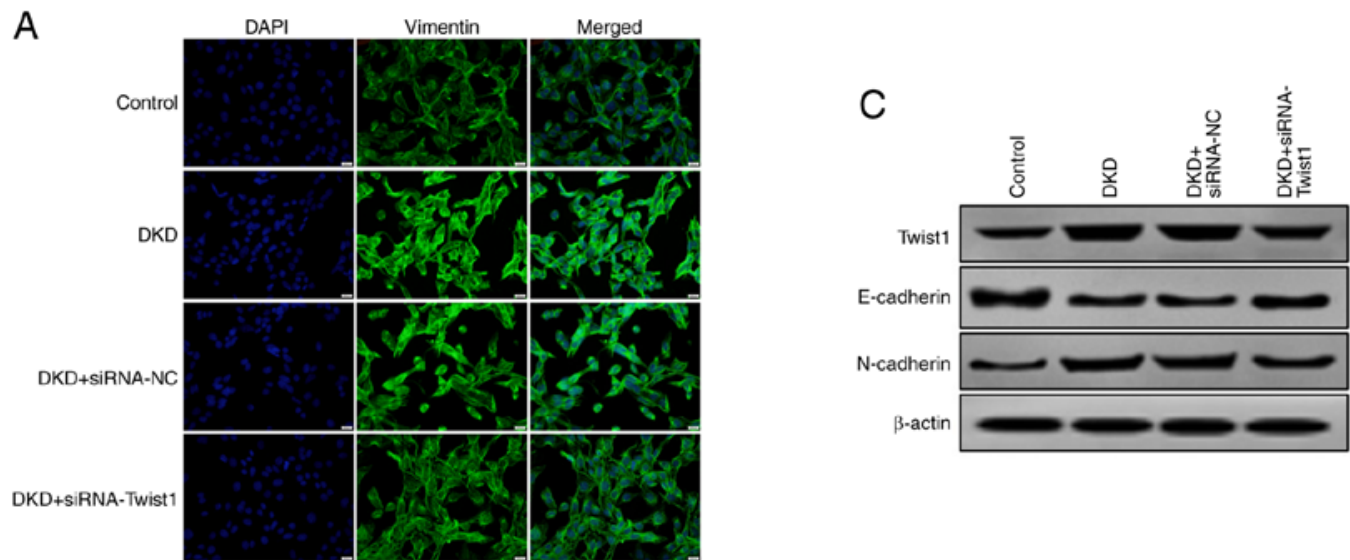

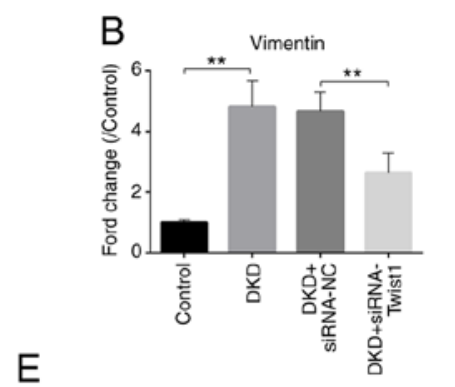

E
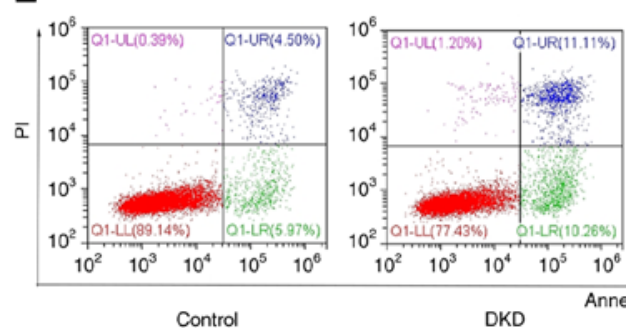
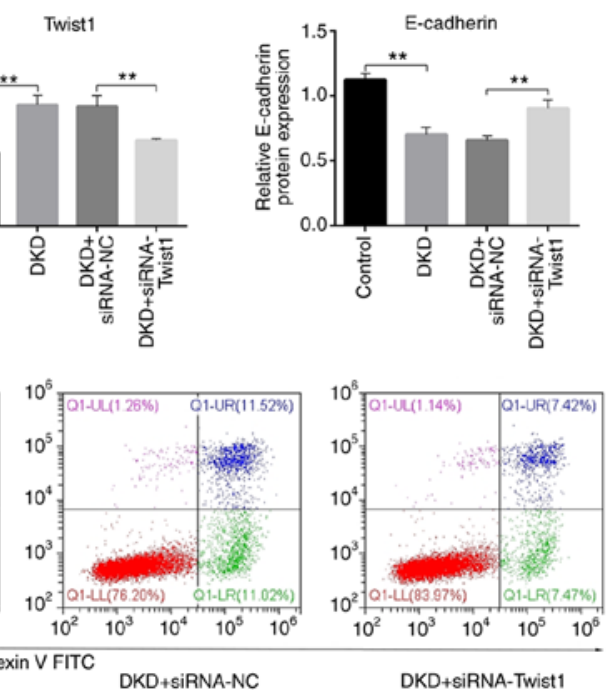

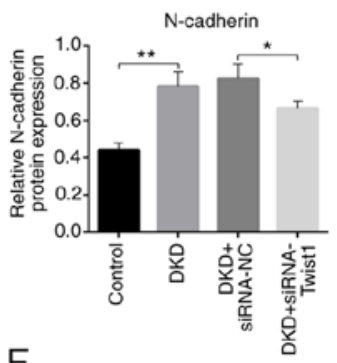

$\mathrm{F}$

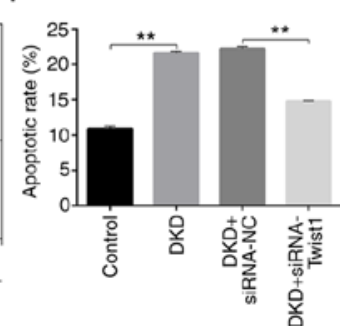

Figure 4. Twist1 interference inhibits epithelial-mesenchymal transition and apoptosis. (A) Immunofluorescence of vimentin expression and localization. (Scale bar, $20 \mu \mathrm{m}$; magnification, x400). (B) Semi-quantitative evaluation of vimentin, as determined by immunofluorescence. Control values were set at 1. (C) Representative western blots of Twist1, N-cadherin and E-cadherin expression in the different groups. (D) Densitometric analysis of Twist1, N-cadherin and E-cadherin expression, as determined by western blotting. (E) Cellular apoptosis, as detected by flow cytometry, in each group. (F) Apoptotic rates in each group. Control, cells treated with serum from control mice (C57BLKS/J db/m) for $24 \mathrm{~h}$; DKD, cells treated with serum from DKD mice (C57BLKS/J $\mathrm{db} / \mathrm{db}$ ) for $24 \mathrm{~h}$; DKD + siRNA-NC, cells treated with serum from DKD mice (C57BLKS/J db/db) for $24 \mathrm{~h}$, and then transfected with siRNA-NC for $48 \mathrm{~h}$; DKD + siRNA-Twist1, cells treated with serum from DKD mice (C57BLKS/J db/db) for $24 \mathrm{~h}$, and then transfected with siRNA-Twist1 for 48 h. Data analysis was performed by one-way ANOVA followed by Tukey's post-hoc test. Data are presented as the mean \pm standard deviation. ${ }^{* *} \mathrm{P}<0.01$, ${ }^{*} \mathrm{P}<0.05$. DKD, diabetic kidney disease; siRNA, small interfering RNA; NC, negative control.

decreased in the DKD + TG group. The fluorescent signals of LC3II were reduced in the DKD group, but increased in the
DKD + TG group (Fig. 7A and B). These results indicated that TG may alleviate EMT and restore autophagy. After addition 
A

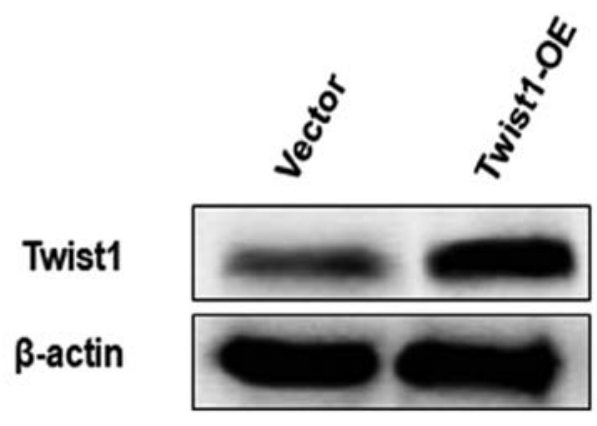

B

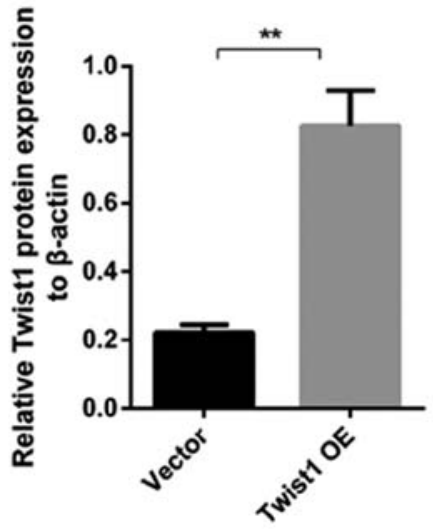

Figure 5. Twist1-OE vector transfection. (A) Representative western blots of Twist1 expression in the different groups. (B) Densitometric analysis of Twist1 expression, as determined by western blotting. Vector, cells transfected with an empty vector; Twist1 OE, cells transfected with the Twist1 OE vector. Data are presented as the mean \pm standard deviation. Data analysis was performed by unpaired Student's t-test. ${ }^{* *} \mathrm{P}<0.01$. OE, overexpression.
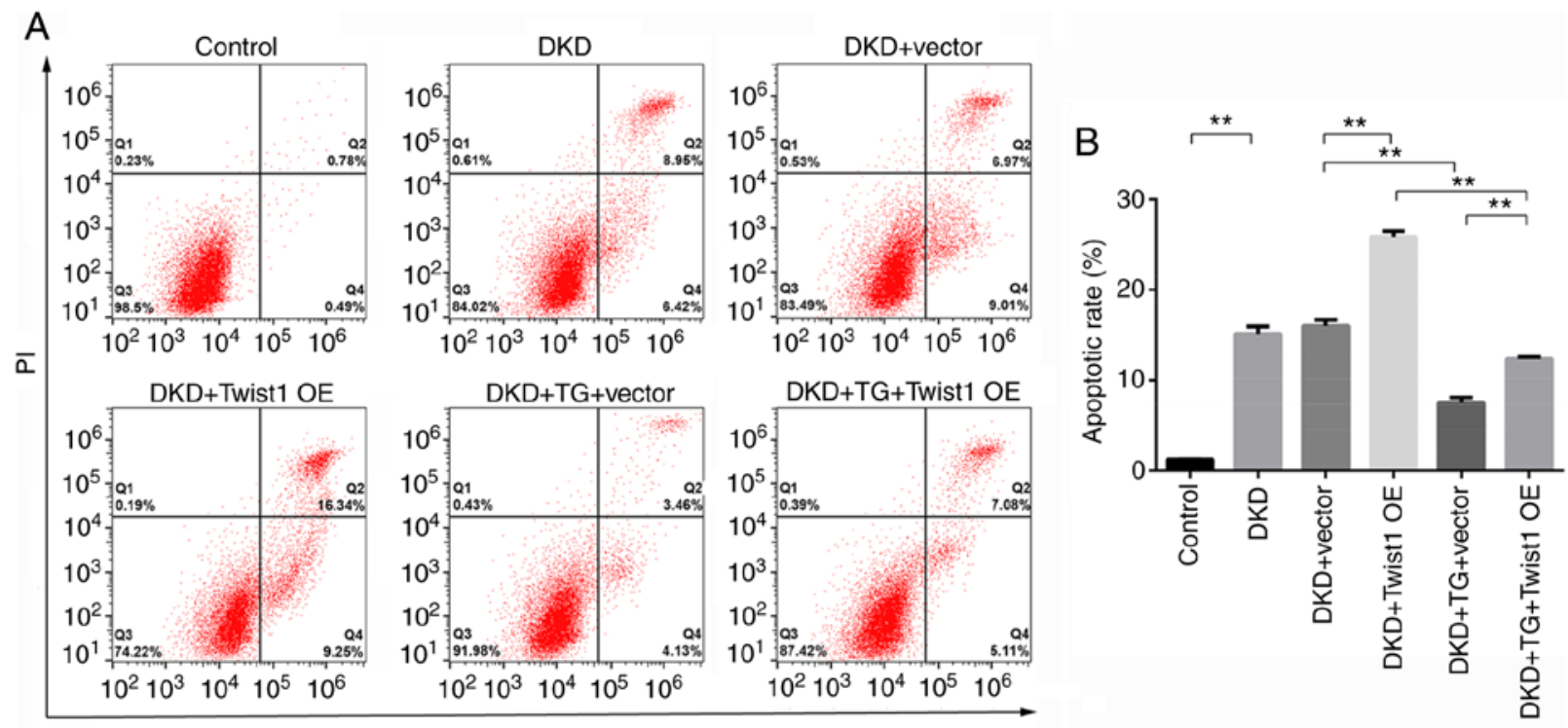

Annexin V FITC

Figure 6. TG affects podocyte apoptosis through Twist1. (A) Cellular apoptosis, as detected by flow cytometry, in each group. (B) Apoptotic rates in each group. Control, cells treated with serum from control mice (C57BLKS/J db/m) for $24 \mathrm{~h}$; DKD, cells treated with serum from DKD mice (C57BLKS/J db/db) for $24 \mathrm{~h}$; DKD + Vector, cells treated with serum from DKD mice (C57BLKS/J db/db) for $24 \mathrm{~h}$, and then transfected with an empty vector for $72 \mathrm{~h}$; DKD + Twist1 OE, cells treated with serum from DKD mice (C57BLKS/J db/db) for $24 \mathrm{~h}$, and then transfected with the Twist1 OE vector for $72 \mathrm{~h}$; DKD + TG + Vector, cells treated with serum from DKD mice (C57BLKS/J db/db) for $24 \mathrm{~h}$ and $1.25 \mu \mathrm{g} / \mathrm{ml} \mathrm{TG}$ for $72 \mathrm{~h}$, and then transfected with an empty vector for $72 \mathrm{~h}$; DKD $+\mathrm{TG}+$ Twist1 OE, cells treated with serum from DKD mice (C57BLKS/J db/db) for $24 \mathrm{~h}$ and $1.25 \mu \mathrm{g} / \mathrm{ml} \mathrm{TG}$ for $72 \mathrm{~h}$, and then transfected with the Twist1 OE vector for $72 \mathrm{~h}$. Data are presented as the mean \pm standard deviation. Data analysis was performed by one-way ANOVA followed by Tukey's post-hoc test. ${ }^{* *} \mathrm{P}<0.01$. DKD, diabetic kidney disease; TG, tripterygium glycoside; OE, overexpression.

of the mTOR activator 3BDO, autophagy was inhibited and the effects of TG on EMT were impaired. The results were further verified by western blotting (Fig. 7C and D), which revealed that the expression levels of p62, N-cadherin, Twist1 and the ratio of p-mTOR to mTOR were significantly increased, whereas the expression levels of E-cadherin and the ratio of LC3II to LC3I were significantly decreased in the DKD group compared with those in the control group. By contrast, after TG treatment, the protein expression levels of p62, Twist1, $\mathrm{N}$-cadherin and the ratio of $\mathrm{p}$-mTOR to mTOR were markedly reduced, whereas those of E-cadherin and the ratio of LC3II to LC3I were significantly increased, demonstrating that TG may upregulate autophagy, alleviate EMT and inhibit Twist1 expression. Following the addition of 3BDO, the therapeutic effect of TG was suppressed, indicating that TG may upregulate autophagy through the mTOR signaling pathway to reduce EMT and Twist1 expression. The flow cytometry results (Fig. 7E and F) revealed a significant increase in the podocyte apoptotic rate of the DKD group, which was reversed with TG treatment. Upon addition of 3BDO, a reduction in the protective effects of TG was noted, indicating that TG may prevent podocyte apoptosis by upregulating autophagy through the mTOR signaling pathway.

Collectively, these results suggested that after culturing podocytes with serum from mice with DKD, EMT and apoptosis were significantly increased, whereas autophagic activity was significantly decreased. TG potentially inhibited the occurrence of DKD-induced podocyte EMT and apoptosis 
A

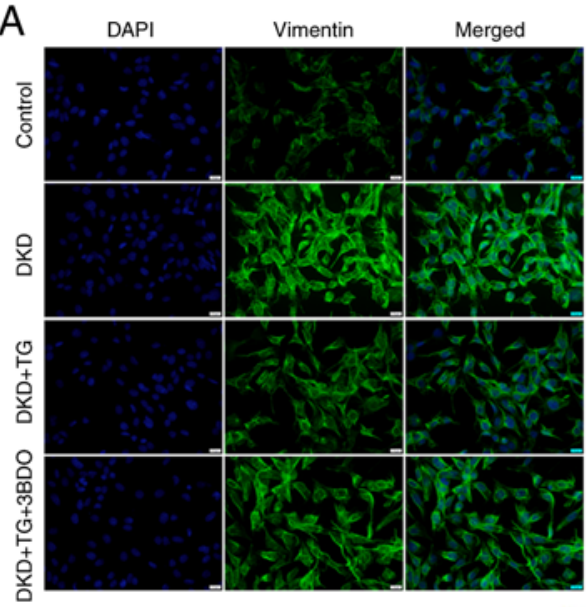

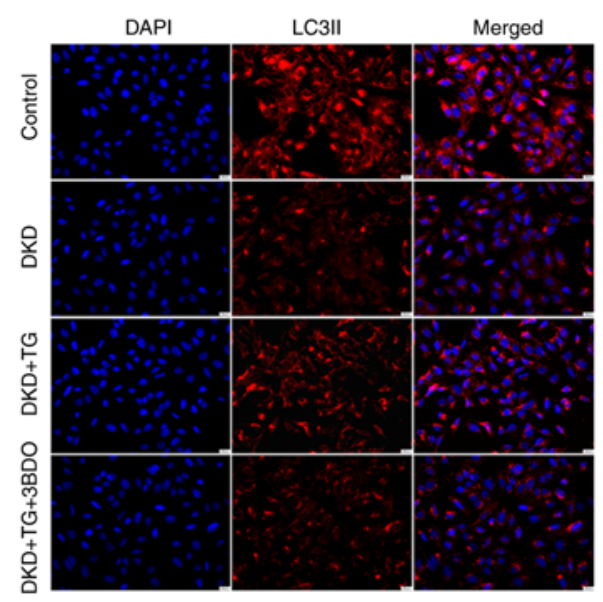
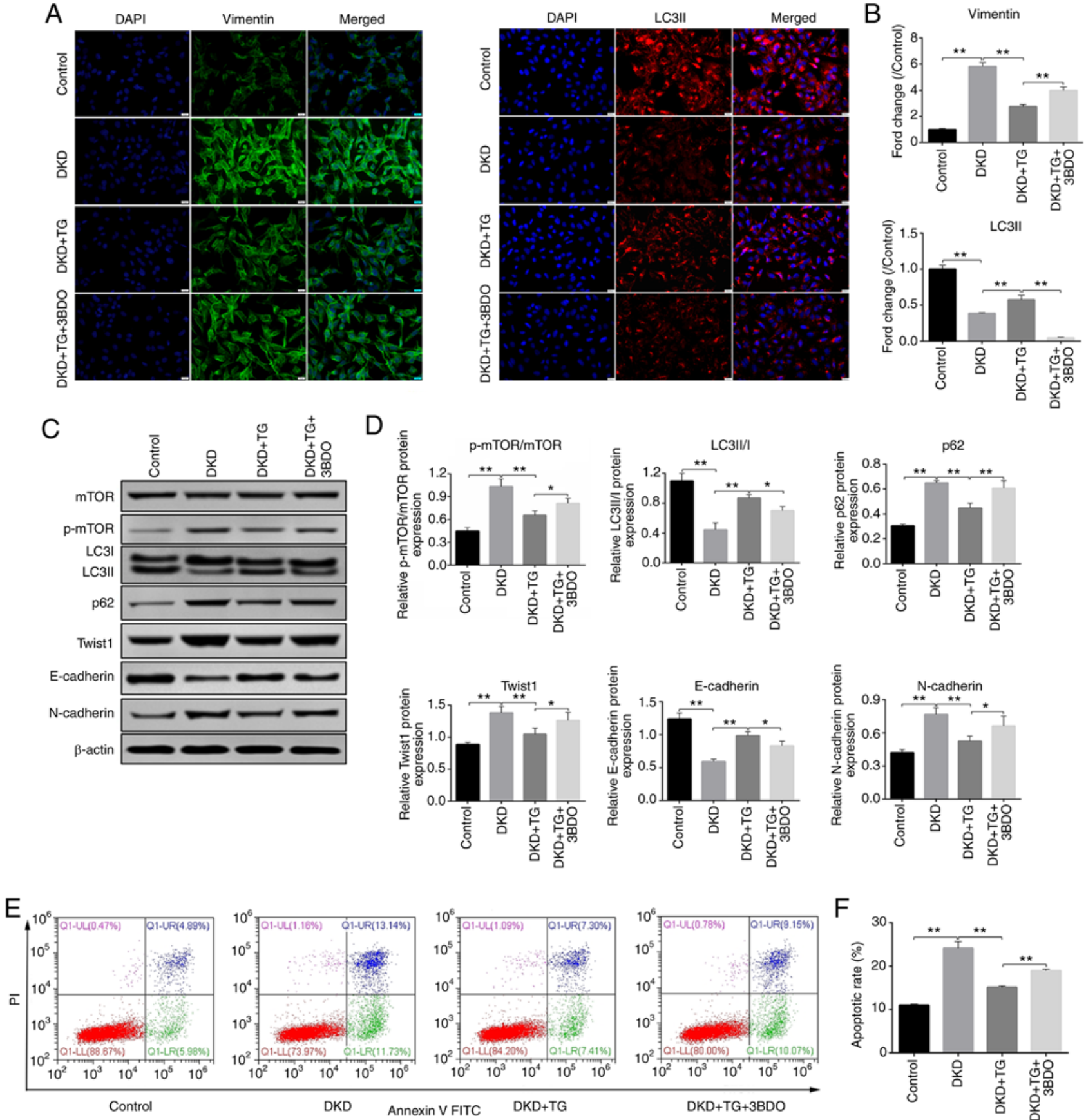

Figure 7. TG inhibits podocyte epithelial-mesenchymal transition and apoptosis through the mTOR/Twistl signaling pathway. (A) Immunofluorescence of vimentin and LC3II (scale bar, $20 \mu \mathrm{m}$; magnification, $\mathrm{x} 400$ ). (B) Semi-quantitative evaluation of vimentin and LC3II, as determined by immunofluorescence. Control values were set at 1. (C) Representative western blots of p-mTOR, LC3, p62, Twist1, E-cadherin and N-cadherin expression in the different groups (D) Densitometric analysis of p-mTOR/mTOR, LC3II/I, p62, Twist1, E-cadherin and N-cadherin expression from different groups, as determined by western blotting. (E) Cellular apoptosis in each group was detected by flow cytometry. (F) Apoptotic rates in each group. Control, cells treated with serum from control mice (C57BLKS/J db/m) for $24 \mathrm{~h}$; DKD, cells treated with serum from DKD mice (C57BLKS/J db/db) for $24 \mathrm{~h}$; DKD + TG, cells treated with serum from DKD mice (C57BLKS/J db/db) for $24 \mathrm{~h}$, and then treated with $1.25 \mu \mathrm{g} / \mathrm{ml}$ TG for $72 \mathrm{~h}$; DKD + TG + 3BDO, cells treated with serum from DKD mice (C57BLKS/J db/db) for $24 \mathrm{~h}$ and $1.25 \mu \mathrm{g} / \mathrm{ml}$ TG for $72 \mathrm{~h}$, and then incubated with $60 \mu \mathrm{M}$ 3BDO for $24 \mathrm{~h}$. Data analysis was performed by one-way ANOVA followed by Tukey's post-hoc test. Data are presented as the mean \pm standard deviation. ${ }^{*} \mathrm{P}<0.05,{ }^{* *} \mathrm{P}<0.01$. TG, tripterygium glycoside; $\mathrm{p}$-, phosphorylated; LC3, microtubule-associated protein 1 light chain 3; DKD, diabetic kidney disease; 3BDO, 3-benzyl-5-((2-nitrophenoxy) methyl)-dihydrofuran-2(3H)-one.

by downregulating mTOR phosphorylation, which promoted autophagy and regulated Twistl expression.

\section{Discussion}

The role of EMT in renal disease has been intensively investigated in previous years. Multiple studies have examined renal
EMT in different animal models of chronic kidney disease and human kidney biopsies $(28,29)$, and increasing evidence has suggested that EMT may contribute to the development and progression of renal fibrosis in DKD (28,30-33). While autophagy deficiency in podocytes is known to serve a role in promoting EMT $(15,19)$, Li et al (34) reported that autophagy could be restored with TG treatment. The present study 
revealed that $\mathrm{TG}$ provided protection against podocyte EMT induced by serum from mice with DKD, and that this effect was mediated by concomitant activation of autophagy and downregulation of Twistl.

Serum E-cadherin, N-cadherin, vimentin, p62 and Twist1 levels from 20 patients with diabetic nephropathy were measured and compared with levels from healthy controls $(n=10)$. Patients with DKD exhibited decreased autophagy levels (as indicated by p62 expression) and increased EMT levels (as indicated by E-cadherin, $\mathrm{N}$-cadherin and vimentin expression). In addition, the changes in EMT and autophagy level were greater in patients with macroalbuminuria compared with in those with microalbuminuria, which further confirmed the significance of EMT in the progression of DKD.

While intracellular signal transduction pathways, including integrin-linked kinase TGF- $\beta / \mathrm{Smad}$ and $\mathrm{Wnt} / \beta$-catenin signaling pathways, have been widely studied in the regulation of renal EMT (35), relatively few studies have explored the involvement of the EMT-inducing factor Twist1 (32). The present study demonstrated that the levels of Twist1 were significantly increased in the serum of patients with DKD. Furthermore, siRNA2-Twist1 was designed and it was revealed that Twist1 inhibition significantly reduced DKD-induced podocyte EMT and apoptosis. By contrast, Twist1 OE increased podocyte apoptosis. In DKD, EMT may occur as cells attempt to evade apoptosis due to exposure to pathophysiological stimuli. EMT and apoptosis are considered to be the major cause of podocyte injury in DKD (36). Thus, the aforementioned findings indicated that Twist1 may act as an important regulatory molecule involved in podocyte injury in DKD.

TG represents a novel, effective and safe drug for treating DKD in patients with proteinuria (37-39) by targeting pathways associated with renal inflammation and oxidative stress $(38,40)$. Furthermore, TG has been reported as a therapeutic target of podocyte autophagy (26,34,41-44), and autophagy activation was shown to alleviate human podocyte injury induced by high glucose (45). In the present study, the flow cytometry results demonstrated that TG treatment could inhibit podocyte apoptosis by targeting Twist1. Further experiments indicated that TG could regulate Twist1 expression, relieve podocyte EMT and decrease podocyte apoptosis through the autophagy pathway. Autophagy deficiency is known to prevent Twist1 and SQSTM1/p62 degradation via the autophagy pathway, leading to aggregation of Twist1 and SQSTM1/p62. SQSTM1/p62 aggregates and subsequently binds to Twist1, thus preventing its degradation through the ubiquitination pathway, which ultimately promotes EMT (19). These previous findings are consistent with the current observations that Twist 1 expression and EMT levels were increased, but autophagy levels were significantly reduced, in the serum of patients with DKD. Consistent with this, the present study revealed that TG restored podocyte autophagy, which may promote Twistl protein degradation and alleviate EMT.

3BDO, a well-known mTOR activator, attenuated the therapeutic effects of TG, indicating that autophagy is a therapeutic target of TG and that TG restores autophagy via the mTOR signaling pathway. The PI3K/Akt/mTOR signaling pathway has been identified as a critical signaling pathway in the regulation of cellular autophagy and apoptosis, and targeting this signaling pathway has been suggested as a prospective strategy for cancer treatment (46). Furthermore, TG inhibited the proliferation of glomerular mesangial cells to prevent diabetic glomerulosclerosis through the Akt/mTOR signaling pathway (47). The PI3K/Akt/mTOR signaling pathway has also been shown to participate in the regulation of cellular EMT and cellular autophagy $(48,49)$. To determine the contribution of the mTOR signaling pathway in the TG-mediated anti-apoptotic effect on podocytes, the present study examined the phosphorylation and expression of mTOR in podocytes cultured with DKD mice serum. The results revealed that p-mTOR and p62 were upregulated, whereas LC3II was downregulated. Upon TG treatment, autophagy was activated, and significant downregulation of p-mTOR was observed. However, no significant differences in total mTOR expression levels were noticed between the TG-treated and untreated groups. These findings suggested that TG may inhibit the phosphorylation of mTOR while mediating autophagy in podocytes, rather than inhibiting mTOR formation.

Although Twist1 serves a key role in EMT, studies exploring its role in molecular-targeted therapy remain limited. To the best of our knowledge, the present study is the first to confirm the involvement of Twist1 in DKD progression, suggesting that Twistl may have the potential to act as a molecular target for DKD treatment. The present study also demonstrated that TG treatment could inhibit podocyte apoptosis via the Twist1 pathway in DKD. However, the effect of TG targeting the Twist1 pathway on podocyte EMT and autophagy was not explored, and further research is required to clarify the effect of Twist1 on podocyte autophagy regulation.

In conclusion, the present study demonstrated that TG may effectively prevent podocyte EMT in DKD, which was mediated, at least partly, by downregulating mTOR phosphorylation and increasing autophagy, thus resulting in a decrease in Twist1 expression. These findings provide novel insights into the molecular mechanisms underlying the protective effects of TG in DKD.

\section{Acknowledgements}

Not applicable.

\section{Funding}

The present study was supported by grants from the Natural Science Foundation of Zhejiang Province (grant nos. LY16H050005, LQ15H050002 and Y18H050024), the Project of Scientific Research Foundation of Chinese Medicine (grant nos. 2016ZA023 2017ZA008 and 2015ZZ002), and the General Project of the Medical and Health of Zhejiang Province (grant no. 2016KYA015). The funding bodies had no role in the design of the study, or in the collection, analysis and interpretation of the data, or in writing the manuscript.

\section{Availability of data and materials}

The datasets used and/or analyzed during the present study are available from the corresponding author on reasonable request.

\section{Authors' contributions}

QH and JJ designed the present study and provided administrative support. MT collected samples and clinical information. 
XL, DW, KH, DZ and MT analyzed and interpreted the data. MT and JJ were involved in drafting the manuscript. QH and JJ confirm the authenticity of all the raw data. All authors read and approved the final manuscript.

\section{Ethics approval and consent to participate}

The present study involving patient samples was approved by the local Ethics Committee of Zhejiang Provincial People's Hospital (Hangzhou, China). The present study was performed in accordance with the ethical standards of the 1964 Declaration of Helsinki. All the enrolled patients provided written informed consent for renal biopsy and participation in research before the renal biopsy was performed. All protocols involving animals were approved by the Institutional Animal Care and Use Committee of Zhejiang Provincial People's Hospital.

\section{Patient consent for publication}

Not applicable.

\section{Competing interests}

The authors declare that they have no competing interests.

\section{References}

1. Saran R, Robinson B, Abbott KC, Agodoa LYC, Albertus P, Ayanian J, Balkrishnan R, Bragg-Gresham J, Cao J, Chen JL, et al: US Renal Data System 2016 Annual Data Report: epidemiology of kidney disease in the United States. Am J Kidney Dis 69 (Suppl 1) A7-A8, 2017.

2. Matoba K, Takeda Y, Nagai Y, Kawanami D, Utsunomiya K and Nishimura R: Unraveling the role of inflammation in the pathogenesis of diabetic kidney disease. Int J Mol Sci 20: 3393, 2019.

3. Stitt-Cavanagh E, MacLeod L and Kennedy C: The podocyte in diabetic kidney disease. ScientificWorldJournal 9: 1127-1139, 2009.

4. Guo J, Xia N, Yang L, Zhou S, Zhang Q, Qiao Y and Liu Z: GSK-3beta and vitamin $\mathrm{D}$ receptor are involved in beta-catenin and snail signaling in high glucose-induced epithelial-mesenchymal transition of mouse podocytes. Cell Physiol Biochem 33: 1087-1096, 2014

5. Li Y, Kang YS, Dai C, Kiss LP, Wen X and Liu Y: Epitheliato-mesenchymal transition is a potential pathway leading to podocyte dysfunction and proteinuria. Am J Pathol 172: 299-308, 2008.

6. Liu L, Fu W, Xu J, Shao L and Wang Y: Effect of BMP7 on podocyte transdifferentiation and Smad7 expression induced by hyperglycemia. Clin Nephrol 84: 95-99, 2015.

7. Dai HY, Zheng M, Tang RN, Ni J, Ma KL, Li Q and Liu BC: Effects of angiotensin receptor blocker on phenotypic alterations of podocytes in early diabetic nephropathy. Am J Med Sci 341: 207-214, 2011.

8. Kang YS, Li Y, Dai C, Kiss LP, Wu C and Liu Y: Inhibition of integrin-linked kinase blocks podocyte epithelial-mesenchymal transition and ameliorates proteinuria. Kidney Int 78: 363-373, 2010.

9. Jin J, Zhang Z, Chen J, Liu Y, Chen Q and Wang Q: Jixuepaidu Tang-1 inhibits epithelial-mesenchymal transition and alleviates renal damage in DN mice through suppressing long non-coding RNA LOC498759. Cell Cycle 18: 3125-3136, 2019

10. Li CX, Xia M, Han WQ, Li XX, Zhang C, Boini KM, Liu XC and Li PL: Reversal by growth hormone of homocysteine-induced epithelial-to-mesenchymal transition through membrane raft-redox signaling in podocytes. Cell Physiol Biochem 27: 691-702, 2011 .

11. Yamaguchi Y, Iwano M, Suzuki D, Nakatani K, Kimura K, Harada K, Kubo A, Akai Y, Toyoda M, Kanauchi M, et al: Epithelial-mesenchymal transition as a potential explanation for podocyte depletion in diabetic nephropathy. Am J Kidney Dis 54: 653-664, 2009.
12. Doublier S, Salvidio G, Lupia E, Ruotsalainen V, Verzola D, Deferrari G and Camussi G: Nephrin expression is reduced in human diabetic nephropathy: Evidence for a distinct role for glycated albumin and angiotensin II. Diabetes 52: 1023-1030, 2003.

13. Ravikumar B, Sarkar S, Davies JE, Futter M, Garcia-Arencibia M, Green-Thompson ZW, Jimenez-Sanchez M, Korolchuk VI, Lichtenberg M, Luo S, et al: Regulation of mammalian autophagy in physiology and pathophysiology. Physiol Rev 90: 1383-1435, 2010.

14. Yasuda-Yamahara M, Kume S, Tagawa A, Maegawa $\mathrm{H}$ and Uzu T: Emerging role of podocyte autophagy in the progression of diabetic nephropathy. Autophagy 11: 2385-2386, 2015.

15. Li G, Li CX, Xia M, Ritter JK, Gehr TW, Boini K and Li PL: Enhanced epithelial-to-mesenchymal transition associated with lysosome dysfunction in podocytes: role of p62/Sequestosome 1 as a signaling hub. Cell Physiol Biochem 35: 1773-1786, 2015.

16. Shibue T and Weinberg RA: EMT, CSCs, and drug resistance: The mechanistic link and clinical implications. Nat Rev Clin Oncol 14: 611-629, 2017.

17. Meng J, Chen S, Han JX, Qian B, Wang XR, Zhong WL, Qin Y, Zhang $\mathrm{H}$, Gao WF, Lei YY, et al: Twistl regulates vimentin through Cul2 circular RNA to promote EMT in hepatocellular carcinoma. Cancer Res 78: 4150-4162, 2018.

18. Liu W, Zhang B, Xu N, Wang MJ and Liu Q: miR-326 regulates EMT and metastasis of endometrial cancer through targeting TWIST1. Eur Rev Med Pharmacol Sci 21: 3787-3793, 2017.

19. Qiang L and He YY: Autophagy deficiency stabilizes TWIST1 to promote epithelial-mesenchymal transition. Autophagy 10: 1864-1865, 2014

20. Cui J, Chen X and Su JC: Advanced progress of main pharmacology activities of triptolide. Zhongguo Zhongyao Zazhi 42: 2655-2658, 2017 (In Chinese).

21. Zhang H, Sun W, Wan Y, Che X, He F, Pu H and Dou C: Preventive effects of multi-glycoside of Tripterygium wilfordii on glomerular lesions in experimental diabetic nephropathy. Zhongguo Zhongyao Zazhi 35: 1460-1465, 2010 (In Chinese).

22. Ma RX, Zhao N and Zhang W: The effects and mechanism of Tripterygium wilfordii Hook F combination with irbesartan on urinary podocyte excretion in diabetic nephropathy patients. Zhonghua Nei Ke Za Zhi 52: 469-473, 2013 (In Chinese).

23. Sethi S, Haas M, Markowitz GS, D'Agati VD, Rennke HG, Jennette JC, Bajema IM, Alpers CE, Chang A, Cornell LD, et al: Mayo clinic/renal pathology society consensus report on pathologic classification, diagnosis, and reporting of GN. J Am Soc Nephrol 27: 1278-1287, 2016.

24. de Cabo R, Fürer-Galbán S, Anson RM, Gilman C, Gorospe M and Lane MA: An in vitro model of caloric restriction. Exp Gerontol 38: 631-639, 2003

25. National Research Council Committee for the Update of the Guide for the $\mathrm{C}$ and Use of Laboratory A: The National Academies Collection: Reports funded by National Institutes of Health. In: Guide for the Care and Use of Laboratory Animals National Academies Press Copyright ${ }^{\mathscr{O}}$ 2011. National Academy of Sciences, Washington, DC, 2011.

26. Zhan H, Jin J, Liang S, Zhao L, Gong J and He Q: Tripterygium glycoside protects diabetic kidney disease mouse serum-induced podocyte injury by upregulating autophagy and downregulating $\beta$-arrestin-1. Histol Histopathol 34: 943-952, 2019.

27. Xia M, Conley SM, Li G, Li PL and Boini KM: Inhibition of hyperhomocysteinemia-induced inflammasome activation and glomerular sclerosis by NLRP3 gene deletion. Cell Physiol Biochem 34: 829-841, 2014.

28. Rastaldi MP, Ferrario F, Giardino L, Dell'Antonio G, Grillo C, Grillo P, Strutz F, Müller GA, Colasanti G and D'Amico G: Epithelial-mesenchymal transition of tubular epithelial cells in human renal biopsies. Kidney Int 62: 137-146, 2002.

29. Zeisberg M and Kalluri R: The role of epithelial-to-mesenchymal transition in renal fibrosis. J Mol Med (Berl) 82: 175-181, 2004.

30. Reidy K and Susztak K: Epithelial-mesenchymal transition and podocyte loss in diabetic kidney disease. Am J Kidney Dis 54: 590-593, 2009.

31. Srivastava SP, Koya D and Kanasaki K: MicroRNAs in kidney fibrosis and diabetic nephropathy: Roles on EMT and EndMT. BioMed Res Int 2013: 125469, 2013.

32. Loeffler I and Wolf G: Epithelial-to-mesenchymal transition in diabetic nephropathy: Fact or Fiction? Cells 4: 631-652, 2015.

33. Hills CE and Squires PE: The role of TGF- $\beta$ and epithelial-to mesenchymal transition in diabetic nephropathy. Cytokine Growth Factor Rev 22: 131-139, 2011. 
34. Li XY, Wang SS, Han Z, Han F, Chang YP, Yang Y, Xue M, Sun B and Chen LM: Triptolide restores autophagy to alleviate diabetic renal fibrosis through the miR-141-3p/PTEN/Akt/mTOR pathway. Mol Ther Nucleic Acids 9: 48-56, 2017.

35. Liu Y: New insights into epithelial-mesenchymal transition in kidney fibrosis. J Am Soc Nephrol 21: 212-222, 2010.

36. Dai H, Liu Q and Liu B: Research progress on mechanism of podocyte depletion in diabetic nephropathy. J Diabetes Res 2017: 2615286, 2017.

37. Wang J, Chen N, Fang L, Feng Z, Li G, Mucelli A, Zhang X and Zhou X: A systematic review about the efficacy and safety of Tripterygium wilfordii Hook.f. preparations used for the management of rheumatoid arthritis. Evid Based Complement Alternat Med 2018: 1567463, 2018.

38. Gao Q, Shen W, Qin W, Zheng C, Zhang M, Zeng C, Wang S, Wang J, Zhu X and Liu Z: Treatment of db/db diabetic mice with triptolide: A novel therapy for diabetic nephropathy. Nephrol Dial Transplant 25: 3539-3547, 2010.

39. Ge Y, Xie H, Li S, Jin B, Hou J, Zhang H, Shi M and Liu Z: Treatment of diabetic nephropathy with Tripterygium wilfordi Hook F extract: A prospective, randomized, controlled clinical trial. J Transl Med 11: 134-134, 2013.

40. Guo H, Pan C, Chang B, Wu X, Guo J, Zhou Y, Liu H, Zhu Z, Chang B and Chen L: Triptolide Improves Diabetic Nephropathy by Regulating Th Cell Balance and Macrophage Infiltration in Rat Models of Diabetic Nephropathy. Exp Clin Endocrinol Diabetes 124: 389-398, 2016.

41. Chen ZH, Qin WS, Zeng CH, Zheng CX, Hong YM, Lu YZ, Li LS and Liu ZH: Triptolide reduces proteinuria in experimental membranous nephropathy and protects against C5b-9-induced podocyte injury in vitro. Kidney Int 77: 974-988, 2010.

42. Gong J, Jin J, Zhao L, Li Y, Li Y and He Q: Tripterygium glycoside protects against puromycin amino nucleoside-induced podocyte injury by upregulating autophagy. Int J Mol Med 42: 115-122, 2018
43. Chan SF, Chen YY, Lin JJ, Liao CL, Ko YC, Tang NY, Kuo CL, Liu KC and Chung JG: Triptolide induced cell death through apoptosis and autophagy in murine leukemia WEHI-3 cells in vitro and promoting immune responses in WEHI-3 generated leukemia mice in vivo. Environ Toxicol 32: 550-568, 2017.

44. Zhao F, Huang W, Zhang Z, Mao L, Han Y, Yan J and Lei M: Triptolide induces protective autophagy through activation of the CaMKK $\beta$-AMPK signaling pathway in prostate cancer cells Oncotarget 7: 5366-5382, 2016

45. Xin W, Li Z, Xu Y, Yu Y, Zhou Q, Chen L and Wan Q: Autophagy protects human podocytes from high glucose-induced injury by preventing insulin resistance. Metabolism 65: 1307-1315, 2016.

46. Rodon J, Dienstmann R, Serra V and Tabernero J: Development of PI3K inhibitors: Lessons learned from early clinical trials. Nat Rev Clin Oncol 10: 143-153, 2013.

47. Han F, Xue M, Chang Y, Li X, Yang Y, Sun B and Chen L: Triptolide suppresses glomerular mesangial cell proliferation in diabetic nephropathy is associated with inhibition of PDK1/Akt/ mTOR pathway. Int J Biol Sci 13: 1266-1275, 2017.

48. Lamouille S and Derynck R: Cell size and invasion in TGF-beta-induced epithelial to mesenchymal transition is regulated by activation of the mTOR pathway. J Cell Biol 178: 437-451, 2007.

49. Wang F, Li H, Yan XG, Zhou ZW, Yi ZG, He ZX, Pan ST, Yang YX, Wang ZZ, Zhang X, et al: Alisertib induces cell cycle arrest and autophagy and suppresses epithelial-to-mesenchymal transition involving PI3K/Akt/mTOR and sirtuin 1-mediated signaling pathways in human pancreatic cancer cells. Drug Des Devel Ther 9: 575-601, 2015

This work is licensed under a Creative Commons Attribution-NonCommercial-NoDerivatives 4.0 International (CC BY-NC-ND 4.0) License. 